\title{
Household transmissibility of avian influenza A (H7N9) virus, China, February to May 2013 and October 2013 to March 2014
}

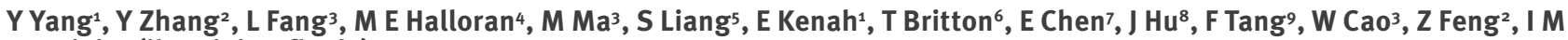

Longini Jr (ilongini@ufl.edu) ${ }^{1}$

1. Department of Bíostatistics and Emerging Pathogens Institute, University of Florida, Gainesville, Florida, United States

2. Public Health Emergency Center, Chinese Center for Disease Prevention and Control, Beijing, China

3. State Key Laboratory of Pathogen and Biosecurity, Beijing Institute of Microbiology and Epidemiology, Beijing, China

4. Vaccine and Infectious Diseases Division, Fred Hutchinson Cancer Research Center, Seattle, Washington, United States

5. Department of Environmental and Global Health, University of Florida, Gainesville, Florida, United States

6. Department of Mathematics, Stockholm University, Stockholm, Sweden

7. Provincial Center for Disease Prevention and Control, Hangzhou, Zhejiang, China

8. Municipal Center for Disease Prevention and Control, Shanghai, China

9. Provincial Center for Disease Prevention and Control, Nanjing, Jiangsu, China

Citation style for this article:

Yang Y, Zhang Y, Fang L, Halloran ME, Ma M, Liang S, Kenah E, Britton T, Chen E, Hu J, Tang F, Cao W, Feng Z, Longini Jr IM. Household transmissibility of avian influenza $\mathrm{A}(\mathrm{H} 7 \mathrm{Ng})$ virus, China, February to May 2013 and October 2013 to March 2014. Euro Surveill. 2015;20(10):pii=21056. Available online: http://www.

eurosurveillance.org/ViewArticle.aspx?Articleld $=21056$

Article submitted on 19 August 2014 / published on 12 March 2015

To study human-to-human transmissibility of the avian influenza $\mathrm{A}\left(\mathrm{H}_{7} \mathrm{Ng}\right)$ virus in China, household contact information was collected for 125 index cases during the spring wave (February to May 2013), and for 187 index cases during the winter wave (October 2013 to March 2014). Using a statistical model, we found evidence for human-to-human transmission, but such transmission is not sustainable. Under plausible assumptions about the natural history of disease and the relative transmission frequencies in settings other than household, we estimate the household secondary attack rate (SAR) among humans to be $1.4 \%$ (95\% $\mathrm{Cl}: 0.8$ to 2.3), and the basic reproductive number $R_{0}$ to be 0.08 ( $95 \% \mathrm{Cl}: 0.05$ to 0.13$)$. The estimates range from $1.3 \%$ to $2.2 \%$ for SAR and from 0.07 to 0.12 for $R_{0}$ with reasonable changes in the assumptions. There was no significant change in the human-to-human transmissibility of the virus between the two waves, although a minor increase was observed in the winter wave. No sex or age difference in the risk of infection from a human source was found. Human-to-human transmissibility of $\mathrm{H}_{7} \mathrm{~N}_{9}$ continues to be limited, but it needs to be closely monitored for potential increase via genetic reassortment or mutation.

\section{Introduction}

Influenza $\mathrm{A}\left(\mathrm{H}_{7} \mathrm{~N} 9\right)$ was first detected among humans in eastern China in February 2013 and, as of 7 December 2014, had caused 453 laboratory-confirmed clinical human infections with 178 deaths in China, according to the Chinese Center for Disease Control and Prevention (Chinese $C D C$ ). The virus is a reassortant of avian $\mathrm{H}_{7}$, $\mathrm{N}_{9}$ and $\mathrm{H}_{9} \mathrm{~N}_{2}$ strains [1] with evidence of the capacity to bind to mammalian cells $[2,3]$ and limited airborne transmission in animal models [4,5]. Currently, the virus is not pathogenic in birds, but highly pathogenic and virulent in humans [6-9]. Normally, the influenza season in humans in the northern hemisphere runs from October to April with some variation in timing $[10,11]$. After a relative hiatus in the number of human $\mathrm{H}_{7} \mathrm{~N} 9$ cases over the summer of 2013 in China (e.g. only two cases reported in July 2013), a second large wave of cases appeared, starting in October 2013 [12].

While the ongoing sporadic reporting of cases (per communication with the Chinese CDC) implies the $\mathrm{H}_{7} \mathrm{~N} 9$ virus has not yet reached the stage of efficient human-to-human transmission, an animal model has shown another $\mathrm{H}_{7}$ virus was able to transmit among co-housed ferrets without much loss of virulence, with a few mutations obtained after 10 serial passages [13]. It is therefore highly relevant to assess the risks of human-to-human transmission using available data.

The household is a setting well suited to investigating human-to-human transmission of many infectious agents, including influenza viruses [14]. So far, no quantitative analysis of household transmission of $\mathrm{H}_{7} \mathrm{~N} 9$ virus has been reported, though qualitative epidemiological descriptions of possible transmissions in a few families with more than one laboratory-confirmed case have been presented [15-18]. We use household information on laboratory-confirmed cases collected by the Chinese CDC to estimate the household secondary attack rate (SAR), i.e. the probability that a typical index case infects a given household member. In addition, the basic reproductive number, $R_{0}$, defined as the number of people a typical infected person would infect in a completely susceptible population, is estimated from the SAR in conjunction with assumptions about the contribution of schools and the general community relative to households in future epidemics. Other investigators estimated $R_{\mathrm{o}}$ to be 0.10 using the sequence of reported cases during the spring outbreak of 2013 [19]. We provide the first rigorous evaluation of $R_{\mathrm{o}}$ based on transmissibility in the household setting. 
Assumptions about probability distributions for the incubation period and relative infectiousness since symptom onset (day 1) for the infectious period for avian influenza A(H7N9) in human cases

\begin{tabular}{|c|c|c|c|c|c|c|c|c|c|c|c|c|c|}
\hline \multirow{2}{*}{ Duration } & \multicolumn{12}{|c|}{ Probability distributions for the incubation period in days } & \multirow{2}{*}{ Mean } \\
\hline & 1 & 2 & 3 & 4 & 5 & 6 & 7 & 8 & 9 & 10 & 11 & 12 & \\
\hline Short & 0.1 & 0.2 & 0.3 & 0.25 & 0.1 & 0.05 & - & - & - & - & - & - & 3.2 \\
\hline Medium & - & 0.05 & 0.1 & 0.2 & 0.3 & 0.2 & 0.1 & 0.05 & - & - & - & - & 5 \\
\hline Long & - & 0.025 & 0.025 & 0.05 & 0.1 & 0.15 & 0.3 & 0.15 & 0.1 & 0.05 & 0.025 & 0.025 & 7 \\
\hline \multirow{2}{*}{ Duration } & \multicolumn{12}{|c|}{ Relative infectiousness since symptom onset (day 1 ) for the infectious period in days } & $\pi$ \\
\hline & $1-3$ & 4 & 5 & 6 & 7 & 8 & 9 & 10 & 11 & 12 & 13 & 14 & - \\
\hline Short & 1 & 0.8 & 0.6 & 0.3 & 0.1 & - & - & - & - & - & - & - & - \\
\hline Medium & 1 & 1 & 0.9 & 0.8 & 0.7 & 0.5 & 0.3 & 0.1 & - & - & - & - & - \\
\hline Long & 1 & 1 & 1 & 1 & 0.9 & 0.8 & 0.7 & 0.6 & 0.5 & 0.3 & 0.2 & 0.1 & - \\
\hline
\end{tabular}

These assumptions are used in all our subsequent analyses.

\section{Methods}

\section{Household study}

Contact data were collected by the Chinese CDC on households with laboratory-confirmed symptomatic cases over the two distinct waves of the epidemic, 125 households during the first wave in the spring of 2013, and another 187 households from the second wave in the winter and spring of 2013-14, offering a unique opportunity to examine the potential change in humanto-human transmissibility of the virus. This investigation was part of the public health emergency response of Chinese CDC, and therefore no informed consent of the household members was required.

A household in this study was defined as a group of related family members living in the same building structure and in daily close contact with each other (our definition of close contact is given below). The case definitions for clinical and confirmed human infections with $\mathrm{H}_{7} \mathrm{~N}_{9}$ were similar to the $\mathrm{H}_{5} \mathrm{~N}_{1}$ case definitions suggested by the World Health Organization in 2006. Data of both laboratory-confirmed clinical cases and their close contacts, including household members, were obtained from a review of medical records and interviews with relatives, contacts and health-care providers. Close contacts of a confirmed case were monitored for seven days for symptoms, and throat swabs were collected from contacts with respiratory symptoms for laboratory testing. Throat swabs were also collected from some close contacts without symptoms. Details of the case definition, laboratory methods and data collection were described previously [18].

Households of laboratory-confirmed clinical cases from all 10 provinces (Shanghai, Zhejiang, Jiangsu, Anhui, Jiangxi, Shandong, Beijing, Henan, Hunan and Fujian) affected by the first wave were included. Households from three provinces (Zhejiang, Hunan and Guangdong) affected by the second wave during October 2013 to March, 2014, were added. Age, sex and dates of symptom onset, hospitalisation, death and recovery (if known) were collected for all family members. In addition, the following types of contact with the index case were recorded for each family member: (i) dining together; (ii) living in the same housing unit; (iii) sharing utensils, towels, toys, etc.; (iv) having contact with excreta (excrement and urine) of cases; (v) providing care; (vi) visiting; and (vii) other contacts. We define close contact as all contact types except for (vi) and (vii). Non-close contacts were excluded from our analysis.

\section{Natural history of avian influenza A(H7N9) in human cases \\ There is limited information on the natural history of infection in humans caused by the $\mathrm{H}_{7} \mathrm{~N} 9$ virus, as the exposure dates cannot be clearly identified for most cases. However, to estimate transmission probabili- ties, distributional assumptions often have to be made about the natural history, in particular the distributions of the incubation period (i.e. time from infection to the onset of influenza symptoms), the latent period (i.e. time from infection to the onset of infectiousness) and the infectious period (i.e. time that an infected person is infectious to others). However, there are little data about these periods.}

Incubation and latent periods

Cowling et al. estimated the mean incubation period of $\mathrm{H}_{7} \mathrm{Ng}$ to be 3.1 days [6]. Chen et al. reported a 3-, 6- and 8-day lag between last exposure to live poultry and symptom onset in three patients, an average of 5.8 days [20]. Huang et al. found even longer incubation periods, a median of six days (range: 2-10) among 10 patients with a single self-reported exposure day and 7.5 days among 12 patients with multiple self-reported exposure days [21]. In contrast, former results about historical seasonal influenza suggested average incubation periods of those viruses of around two to three days [22]. 
We consider three possible distributions for our analysis (upper panel of Table 1). For the primary analysis, we adopt a minimum $\left(d_{\min }\right)$ of two days and a maximum $\left(d_{\max }\right)$ of eight days, with a probability mass distribution of $\theta=\left(\theta_{d_{\min }}, \cdots, \theta_{d_{\max }}\right)=(0.05,0.1,0.2,0.3,0.2,0.1,0.05)$. For influenza, the latent period and the incubation period are similar [23], and we assume the two periods overlap.

\section{Infectious period}

While the average time from symptom onset to recovery could be as long as 15 days (based on the surveillance data provided by the Chinese $(D C)$, the actual duration of the infectious period is likely to be much shorter. For seasonal influenza, symptoms usually resolve in a week, and previous studies on seasonal and pandemic influenza $A\left(\mathrm{H}_{1} \mathrm{~N}_{1}\right)$ suggested a likely range of 7-10 days after symptom onset for the viral load to become undetectable [24-26]. Information on pathological and immunological response regarding the avian $\mathrm{H}_{7} \mathrm{~N} 9$ is scarce. In a study of mice infected with $\mathrm{H} 7 \mathrm{~N} 9$ virus isolated from humans and ducks, viral titres were not detectable after day 8 post infection [27].

We explore three settings for the infectious period, each setting given by a fixed duration with decaying relative infectivity (lower panel of Table 1). We assume the infectious period starts from the day of symptom onset (day 1). The medium setting is adopted for the primary analysis, i.e. the duration of the infectious period is $D=10$ days, and the relative infectivity levels are $\eta=\left(\eta_{1}, \cdots, \eta_{10}\right)=(1,1,1,1,0.9,0.8,0.7,0.5,0.3,0.1)$.

This setting gives a mean serial interval of approximately 9.4 days, slightly longer than the crude estimate of 7.5 days (see Results).

\section{Human-to-human transmissibility}

A likelihood-based statistical transmission model is used to test the hypothesis of no human-to-human transmission and to estimate the SAR and $R_{0}[22,28]$. Suppose we observe an epidemic from day 1 to day $T$ among a population of $N$ individuals in $H$ households. Person-to-person transmissibility of the virus is measured by the probability that an infectious person infects his or her susceptible household contact in one day, denoted by $p$. The transmission probability can be adjusted for covariates via a logistic regression model given by $\operatorname{logit}\left(p_{i j t}\right)=\operatorname{logit}(p)+X_{i j t}^{\prime} \beta$, where $X_{i j t}$ is the vector of covariates associated with an infectious person $j$ and a susceptible person $i$ on day $t$. We let $\beta$ be the vector of coefficients, where $e^{\beta}$ is interpreted as the odds ratios. To account for exposure to an environmental reservoir such as poultry and to unknown casual contacts with human sources outside of the household, we assume that a susceptible person is infected by external sources during one day with probability $b$. Suppose that we have observed households ascertained by index cases. Let $h(i)$ be the collection of members of the household of individual $i$, and let $\tilde{t}_{i}$ be the symptom onset day of individual $i$. Then, the probability that susceptible individual $i$ escapes infection during day $t$ is

$$
e_{i}(t)=(1-b) \prod_{j \in h(i), j \neq i}\left[1-p_{i j t} \eta\left(t-\tilde{t}_{j}+1\right)\right],
$$

Where $\eta(\mathrm{k})$ is the relative infectiousness of day $k$ of the infectious period. We define $\tilde{t}_{h(i)}$ to be the collection of observed symptom onset days of all members in the household of individual $i$. Let $\theta(k)$ be the probability that the incubation period is $k$ days. The likelihood contribution of individual $i$ is

$L_{i}\left(b, p \mid \tilde{t}_{h(i)}\right)=\left\{\begin{array}{cc}\prod_{t=1}^{T} e_{i}(t), & \text { escaped, } \\ \sum_{t} \theta\left(\tilde{t}_{i}-t\right)\left[1-e_{i}(t)\right] \prod_{\tau=1}^{t-1} e_{i}(\tau), & \text { infected. }\end{array}\right.$

We embed the same likelihood structure in a resampling-based approach to test the null hypothesis $\mathrm{H}_{\mathrm{o}}: p=0 \quad[29]$.

The SAR over an infectious period of $D$ days is

$\mathrm{SAR}=1-\prod_{d=1}^{D} 1-p \eta(d)$.

The SAR specific to a covariate value $X$ is given by replacing $p$ with logit ${ }^{-1}\left[\operatorname{logit}(p)+X^{\prime} \beta\right]$. To estimate $R_{0}$, the fact that the probability of becoming an index case differs across age groups has to be considered, as it is known that older people have a higher chance of visiting high-risk areas such as live poultry markets $[6,30]$. For a population partitioned into three age groups: $0-19,20-59$ and $\geq 60$ years, the basic reproductive number is given by

$R_{0}=\operatorname{SAR}\left[\pi_{1}\left(n_{1}-1\right)\left(1+\rho_{S}+\rho_{C}\right)+\pi_{2}\left(n_{2}-1\right)\left(1+\rho_{C}\right)+\pi_{3}\left(n_{3}-1\right)\left(1+\rho_{C}\right)\right]$,

Where $\pi_{j}$ is the probability that an index case belongs to age group $j, n_{j}$ the average household size if the index case is from age group $j$, and $\rho_{S}$ and $\rho_{c}$ are the relative contact frequencies of an infectious person in school and the community compared with within household, respectively. The community refers to all places other than households and schools where transmission occurs. Lacking the data to determine the $\rho_{S}$ and $\rho_{C}$ specific to China, we use the information in a previous study of influenza $A\left(\mathrm{H}_{1} \mathrm{~N}_{1}\right)$ pdmog in the United States [22], where the plausible ranges for the local reproductive numbers in households $\left(R_{H}\right)$ and schools $\left(R_{S}\right)$ were $0.6-0.9$ and 2.0-2.5, respectively, and the corresponding range for $\rho_{S}=R_{S} / R_{H}$ was 2-4, which is used in our analysis. Another previous study of influenza pandemics in the United States showed that the community at large generally accounts for somewhat fewer transmissions of influenza virus than within households [14]. The values $0,0.5$ and 1.0 were used for $\rho_{C}$ in the $\mathrm{A}\left(\mathrm{H}_{1} \mathrm{~N}_{1}\right)$ pdmog study [22]. In our analysis for $\mathrm{H}_{7} \mathrm{~N} 9$, we use $0.5,1.0$ and 1.5 for $\rho_{C}$ to reflect the higher population density in public places in China.

We estimate $\pi_{j}$ using a conditional Poisson model, in which temporal and spatial heterogeneity are controlled for by aggregating the data by appropriate location and time, e.g. by prefecture and week. Let 
Laboratory-confirmed avian influenza A(H7N9) cases in humans from (A) February-May 2013 (125 households, 130 cases) and (B) October 2013-March 2014 (187 households, 196 cases), household data, China

\section{A. February-May 2013}

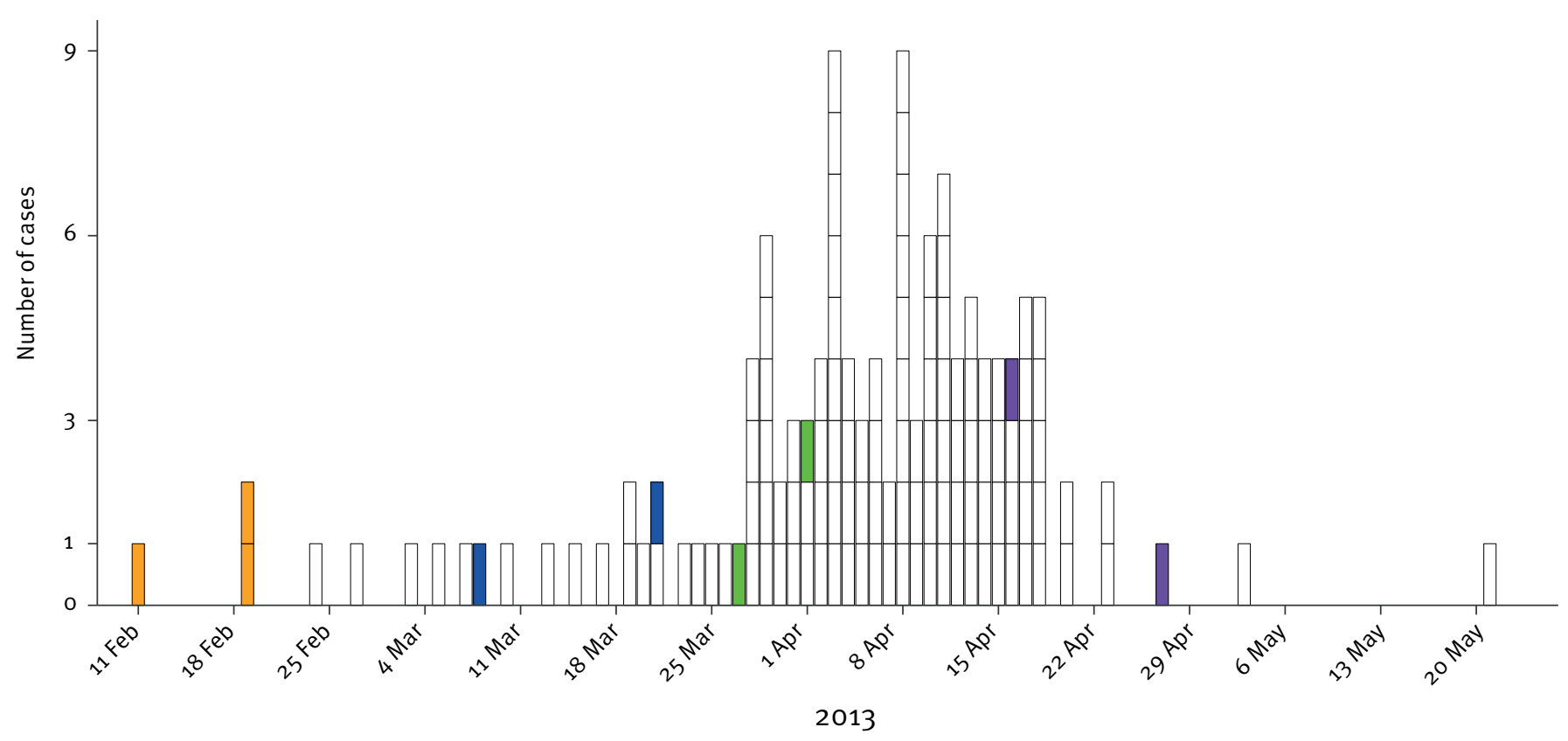

B. October 2013-March 2014

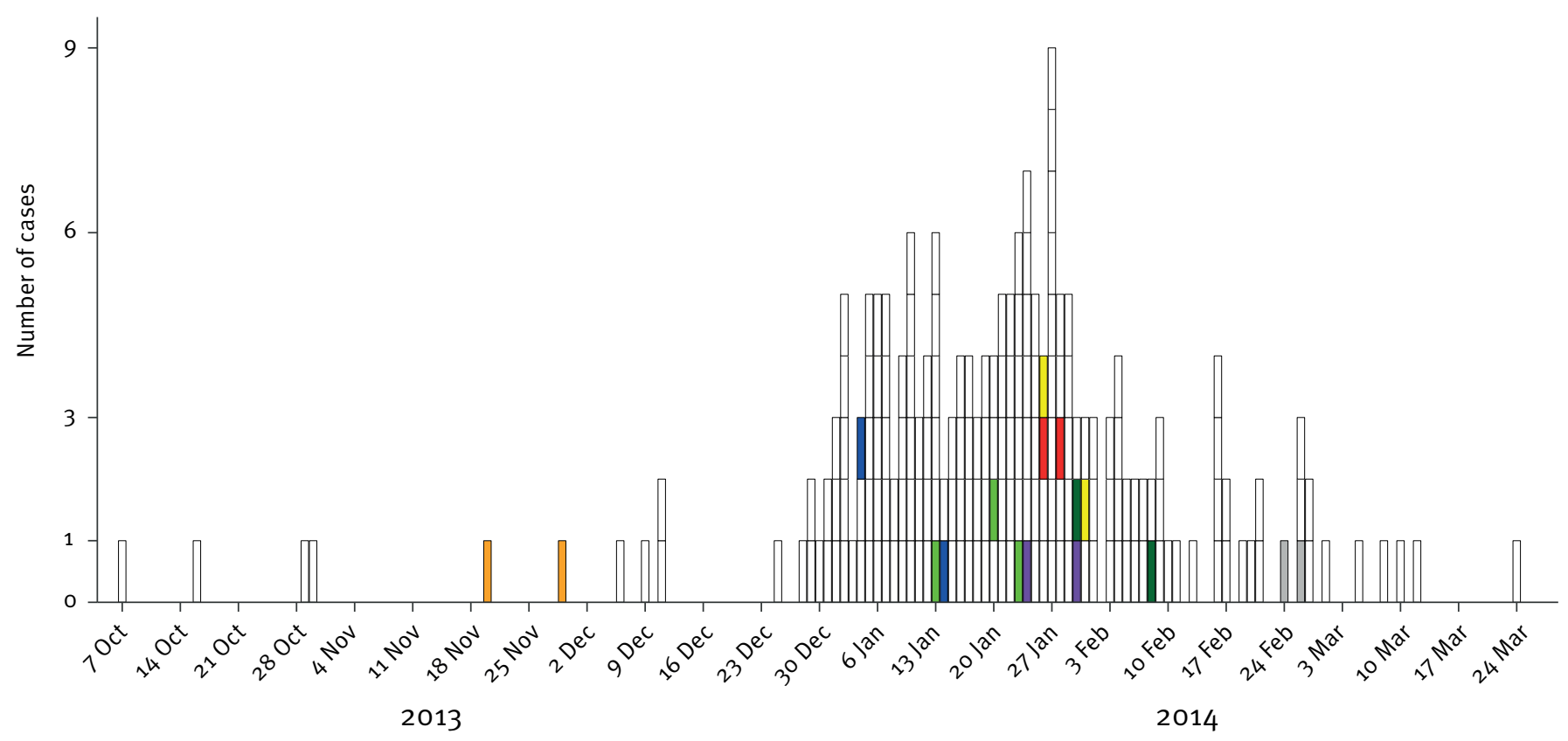

Cases from the same household are shown in the same colour; single index cases are left blank. 


\section{TABLE 2}

Estimates of secondary attack rate and basic reproductive number based on household clusters of avian influenza A(H7N9) cases in humans, ignoring poultry-to-human transmission, China, February-May 2013 (113 households, 118 cases) and October 2013-March 2014 (181 households, 190 cases)

\begin{tabular}{|c|c|c|c|c|c|}
\hline \multirow{3}{*}{$\begin{array}{l}\text { Incubation } \\
\text { period }\end{array}$} & \multirow{3}{*}{$\begin{array}{l}\text { Infectious } \\
\text { period }\end{array}$} & \multirow{3}{*}{ Value } & \multicolumn{3}{|c|}{ Relative contact frequency within hospital vs within household, $\alpha$} \\
\hline & & & 1.0 & 0.5 & 0.1 \\
\hline & & & Estimate $(95 \% \mathrm{Cl})$ & Estimate $(95 \% \mathrm{Cl})$ & Estimate $(95 \% \mathrm{Cl})$ \\
\hline \multirow{9}{*}{ Short } & \multirow{3}{*}{ Short } & SAR & $1.3 \%(0.74$ to 2.2$)$ & $1.4 \%(0.79$ to 2.3$)$ & $1.4 \%$ (o.84 to 2.5$)$ \\
\hline & & $R_{\mathrm{o}}$ & $0.072(0.042$ to 0.12$)$ & $0.076(0.044$ to 0.13$)$ & $0.081(0.047$ to 0.14$)$ \\
\hline & & $\mathrm{p}$ value & 0.16 & - & - \\
\hline & \multirow{3}{*}{ Medium } & SAR & $1.4 \%(0.82$ to 2.3$)$ & $1.6 \%(0.95$ to 2.7$)$ & $1.8 \%(1.1$ to 3.0$)$ \\
\hline & & $R_{\mathrm{o}}$ & 0.078 (0.046 to 0.13$)$ & $0.089(0.053$ to 0.15$)$ & $0.10(0.060$ to 0.17$)$ \\
\hline & & $\mathrm{p}$ value & 0.0005 & - & - \\
\hline & \multirow{3}{*}{ Long } & SAR & $1.4 \%$ (o.83 to 2.4 ) & $1.8 \%(1.0$ to 2.9$)$ & $2.2 \%(1.3$ to 3.7$)$ \\
\hline & & $R_{\mathrm{o}}$ & $0.079(0.047$ to 0.13$)$ & $0.098(0.058$ to 0.17$)$ & 0.12 (0.073 to 0.21$)$ \\
\hline & & $p$ value & 0.0015 & - & - \\
\hline \multirow{9}{*}{ Medium } & \multirow{3}{*}{ Short } & SAR & $1.4 \%$ (o.82 to 2.3 ) & $1.5 \%$ (o.87 to 2.5 ) & $1.6 \%(0.92$ to 2.6$)$ \\
\hline & & $R_{\mathrm{o}}$ & 0.077 (0.046 to 0.13$)$ & $0.082(0.049$ to 0.14$)$ & $0.087(0.052$ to 0.15$)$ \\
\hline & & $p$ value & 0.0175 & - & - \\
\hline & \multirow{3}{*}{ Medium } & SAR & $1.4 \%(0.82$ to 2.3$)$ & $1.6 \%$ (0.95 to 2.7$)$ & $1.8 \%(1.1$ to 3.0$)$ \\
\hline & & $R_{\mathrm{o}}$ & 0.078 (0.046 to 0.13$)$ & $0.090(0.053$ to 0.15$)$ & $0.10(0.060$ to 0.17$)$ \\
\hline & & $\mathrm{p}$ value & 0.0035 & - & - \\
\hline & \multirow{3}{*}{ Long } & SAR & $1.4 \%$ (o.84 to 2.4$)$ & $1.8 \%(1.0$ to 2.9$)$ & $2.2 \%(1.3$ to 3.7$)$ \\
\hline & & $R_{\mathrm{o}}$ & $0.079(0.047$ to 0.13$)$ & $0.098(0.058$ to 0.17$)$ & $0.12(0.073$ to 0.21$)$ \\
\hline & & $\mathrm{p}$ value & 0.020 & - & - \\
\hline \multirow{9}{*}{ Long } & \multirow{3}{*}{ Short } & SAR & $1.4 \%$ (o.82 to 2.3 ) & $1.5 \%$ (o.87 to 2.5 ) & $1.6 \%$ (0.92 to 2.6$)$ \\
\hline & & $R_{0}$ & 0.077 (0.046 to 0.13$)$ & $0.083(0.049$ to 0.14$)$ & $0.087(0.052$ to 0.15$)$ \\
\hline & & $p$ value & 0.047 & - & - \\
\hline & \multirow{3}{*}{ Medium } & SAR & $1.4 \%(0.82$ to 2.3$)$ & $1.6 \%$ (0.95 to 2.7 ) & $1.8 \%(1.1$ to 3.0$)$ \\
\hline & & $R_{\mathrm{o}}$ & 0.078 (0.046 to 0.13$)$ & $0.090(0.053$ to 0.15$)$ & $0.10(0.061$ to 0.17$)$ \\
\hline & & $p$ value & 0.10 & - & - \\
\hline & \multirow{3}{*}{ Long } & SAR & $1.4 \%$ (o.84 to 2.4 ) & $1.8 \%(1.0$ to 2.9$)$ & $2.2 \%(1.3$ to 3.7$)$ \\
\hline & & $R_{0}$ & $0.079(0.047$ to 0.13$)$ & 0.099 (0.058 to 0.17$)$ & $0.12(0.073$ to 0.21$)$ \\
\hline & & $p$ value & 0.44 & - & - \\
\hline
\end{tabular}

$b$ : daily probability of infection by external sources; $\mathrm{Cl}$ : confidence interval; $R_{0}$ : basic reproductive number; $\rho_{c}$ : relative contact frequency in the community; $\rho_{s}$ : relative contact frequency in schools; SAR: secondary attack rate.

Results are based on the assumption of $b=0$ (i.e. ignoring poultry-to-human transmission) and stratified by the duration of the incubation and infectious periods (duration settings are given in Table 1) and the relative contact frequency between a case and their household members within a hospital compared with within the household $(\alpha)$. Relative contact frequencies in schools and the community are set to $\rho_{S}=3$ and $\rho_{C}=1$. The test of $p=0$ (no human-to-human transmission) is performed when $\alpha=1$.

$Y_{k t j}$ be the number of cases in age group $j$ in prefecture $k$ with symptom onset during week $t$. We assume $Y_{k t j} \sim$ Poisson $\left(\lambda_{k t j}\right)$ with the intensity $\lambda_{k t j}=N_{k j} e^{\gamma_{k t}+\beta_{j}}$ where $N_{k j}$ is the population size in age group $j$ of prefecture $k$, and $\exp \left(\gamma_{k t}\right)$ is the rate of a single person in the reference age group of prefecture $k$ becoming a case during week $t$. For the parameters to be identifiable, we set $\beta_{3}=0$, i.e., setting the age group $\geq 60$ years as the reference group. Conditioning on the total number of cases $Y_{k t}=\Sigma_{j} Y_{k t j}$, the vector $\left(Y_{k t 1}, Y_{k t 2}, Y_{k t 3}\right)$ follows a multinomial distribution with size $Y_{k t}$ and probabilities $\pi_{k j}=\frac{N_{k j} e^{\beta j}}{\sum_{l} N_{k l} e^{\beta} l}, j=1,2,3$.
The maximum likelihood estimates of $\beta_{1}$ and $\beta_{2}$, denoted by $\hat{\beta}_{1}$ and $\hat{\beta}_{2}$, are found by maximising the log-likelihood

$\sum_{k, t} \sum_{j=1}^{3} Y_{k t j} \log \left(\pi_{k j}\right)$.

A reasonable estimate for $\pi_{j}$ is

$$
\hat{\pi}_{j}=\frac{N_{j} e^{\widehat{\beta}_{j}}}{\sum_{l} N_{l} e^{\widehat{\beta}_{l}}}
$$

where $N_{j}=\sum_{k} N_{k j}$ and $\hat{\beta}_{3}=0$. Due to data availability, we used surveillance data up to 27 January 2014 to estimate $\pi_{j}$. 


\section{Results}

We collected household data for 130 laboratory-confirmed clinical cases in 125 households in 10 provinces for February to May 2013 and for 196 laboratory-confirmed clinical cases in 187 households in Hunan, Zhejiang and Guangdong provinces for October 2013 to March 2014. The epidemic curves of the cases in these households during the two waves are shown in Figure 1 (panels A and B). Interestingly, multi-case households appeared from the very beginning of the 2013 wave. After excluding households without close contacts, 118 cases in 113 households in the first wave and 190 cases in 181 households in the second were used in this analysis. There was an asymptomatic infection identified in one household in the first wave, but this person was considered a non-case in our analysis; i.e., we only focus on laboratory-confirmed infections with clinical symptoms, because (i) it is not clear whether asymptomatic hosts can transmit the virus or not, and (ii) probably not all asymptomatic infections were detected.

Under the assumption that all non-index cases were infected by the index cases, an estimate of the mean serial interval is 7.5 days ( $95 \%$ confidence interval (CI): 4.9 to 9.0). There was no difference in the distribution of the serial interval between the two waves ( $p$ value $=0.31$ based on the two-sample Wilcoxon test). Using the transmission model, we first test the hypothesis of no human-to-human transmission, and we then estimate the SAR and $R_{0}$ without adjusting for any covariate (Table 2). The estimation of $R_{0}$ is based on the estimates of

$$
\hat{\pi}_{1}=0.044 \text { (95\% Cl: } 0.023 \text { to } 0.083 \text { ), }
$$

$$
\hat{\pi}_{2}=0.44(95 \% \mathrm{Cl}: 0.39 \text { to } 0.51)
$$

and

$$
\left.\hat{\pi}_{3}=0.51 \text { (95\% Cl: } 0.45 \text { to } 0.58\right)
$$

obtained from the conditional Poisson model. In addition to taking into account the uncertainty in the natural history of disease, the results are also stratified by the relative contact frequency between a case and his or her household members within a hospital compared with within the household, denoted by $a$. When $a$ is assumed unknown, its estimate varies dramatically from well below one to well above one, depending on the assumption about the natural history of disease. This indicates a lack of information about $a$ in the data. Consequently, we assume $a$ is known and examine results at three levels of $a: 1.0,0.5$ and 0.1 , based on the rationale that a family member was more likely to be cautious in having contact with an infected person who was hospitalised. Hypothesis testing is performed only under $a=1$. The statistical evidence for humanto-human transmission is significant for most plausible distributions of the incubation and infectious periods except when both of them are short or both are long. The daily probability of infection by external sources, $b$, is estimable only under the setting of both the incubation and infectious periods being short. In all other settings, the maximum likelihood estimate (MLE) of $b$ is given by the boundary value 0 . As a result, we set $b=0$ for estimating SAR and $R_{0}$ in the primary analysis. Given $a=1$, regardless of the assumption of the natural history, the estimates for SAR and $R_{0}$ are generally low and stable. Using median incubation and infectious periods as the primary setting, we estimate SAR as 1.4\% (95\% Cl: 0.8 to 2.3 ) and $R_{\mathrm{o}}$ as 0.08 (95\% Cl: 0.05 to 0.13 ), respectively, i.e. the probability that an $\mathrm{H}_{7} \mathrm{~N} 9$ case infects another household contact is 0.014 , and an $\mathrm{H}_{7} \mathrm{~N} 9$ case infects an average of 0.08 other people. Lower values of $a$ correspond to slightly higher estimates for SAR and $R_{0}$, and the estimates appear higher with longer infectious periods but are insensitive to the incubation period. With $a=0.1$ and a long infectious period, the estimates reach $2.2 \%$ for SAR and 0.12 for $R_{0}$. The interpretation of the estimates for SAR and $R_{0}$ under $a<1$ is limited to the household setting, i.e. as if the infected person will not be hospitalised.

The above results assume the relative contact frequencies in schools and the community are fixed at $\rho_{S}=3$ and $\rho_{C}=1$. A sensitivity analysis for $R_{0}$ with respect to $\rho_{s}$ and $\rho_{C}$ is shown in Figure 2, where three levels chosen from previous work [22] are considered for each parameter. These estimates are not sensitive to $\rho_{s}$, but are sensitive to $\rho_{c}$, which is likely due to the fact that $\rho_{S}$ is only associated with children and children are the least exposed to the environmental reservoir as compared with other age groups. Under long incubation and infectious periods and $a=0.1, \rho_{C}=1.5$ could yield estimates as high as 0.15 for $R_{0}$. On the other hand, under short incubation and infectious periods, $a=1$, and $\rho_{C}=0.5$, the estimate for $R_{0}$ could be as low as 0.05 . Overall, $R_{0}$ is well below one, so that sustained person-to-person transmission will not take place.

With limited data about secondary transmission, we examine whether the household transmissibility of the virus changed between the two waves. In addition, possible heterogeneity in the risk of infection from a human source between the sexes and between age groups (adults (18 years or older) versus children (under 18 years)) are tested using the logistic regression in the chain binomial model. The results are summarised in Table 3, suggesting that the estimated odds ratios of within-household secondary transmission are relatively robust to assumptions about the natural history of the disease and the relative contact frequency in the hospital versus in the household. None of these factors have a significant effect on secondary transmission, possibly due to the lack of a sufficient number of secondary transmissions. However, males appeared to have a somewhat higher risk of infection from a human source compared with females, the odds ratio being 2.24 ( $95 \% \mathrm{Cl}: 0.69$ to 7.26 ), though not statistically significant, under a medium length of incubation and infectious periods and $a=1$. There was no significant difference in the risk between adults and children, the odds ratio being 0.66 ( $95 \% \mathrm{Cl}: 0.20$ to 2.19 ). The odds ratio between the second and the first waves was 1.3 (95\% Cl: 0.4 to 4.27 ), suggesting the virus did not 
Stratified estimates of basic reproductive number based on household clusters of avian influenza A(H7N9) cases in humans, China, February-May 2013 (113 households, 118 cases) and October 2013-March 2014 (181 households, 190 cases)
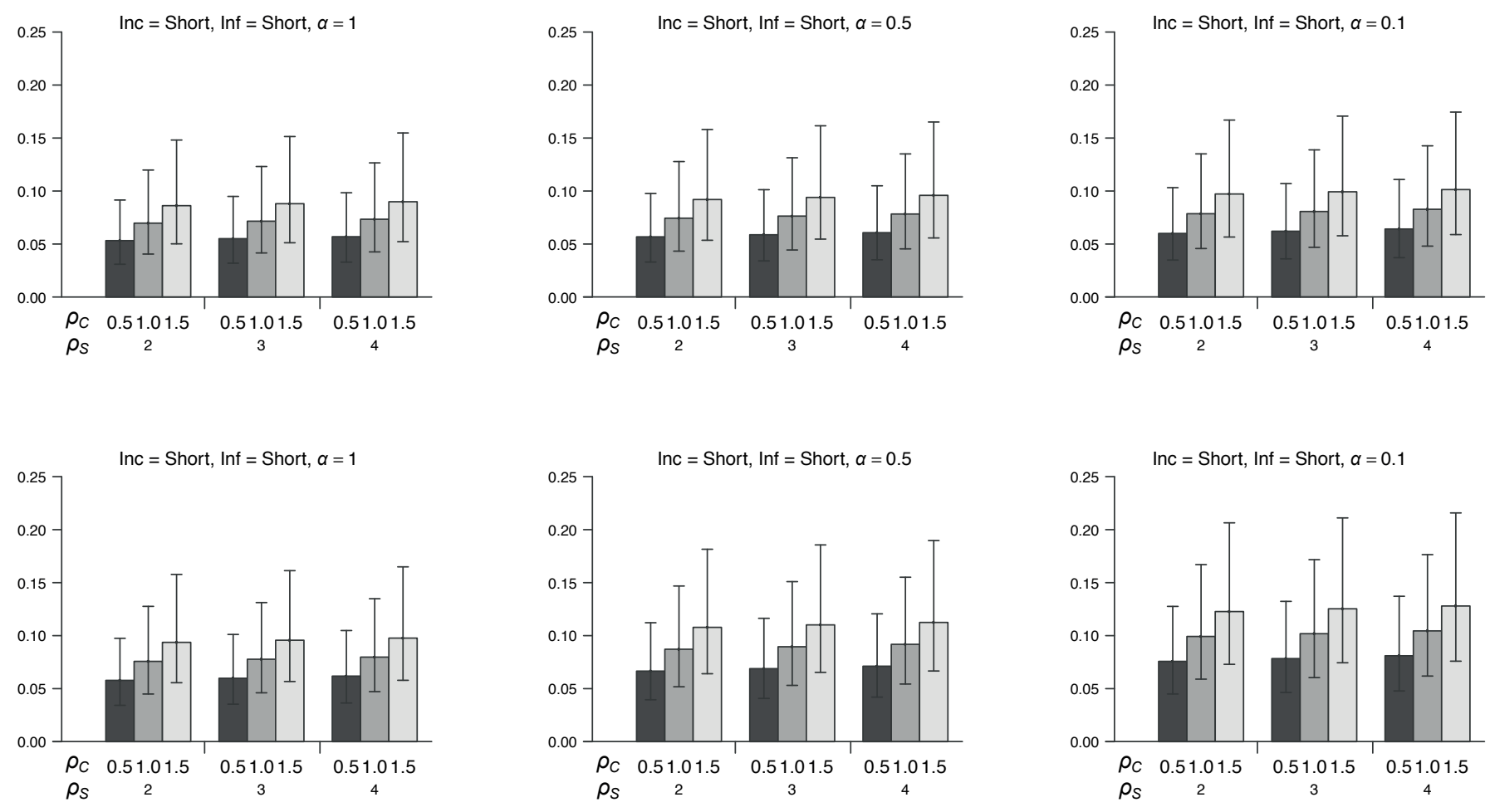

Inc $=$ Short, Inf $=$ Short, $\alpha=1$

$\operatorname{Inc}=$ Short, Inf $=$ Short, $\alpha=0.5$
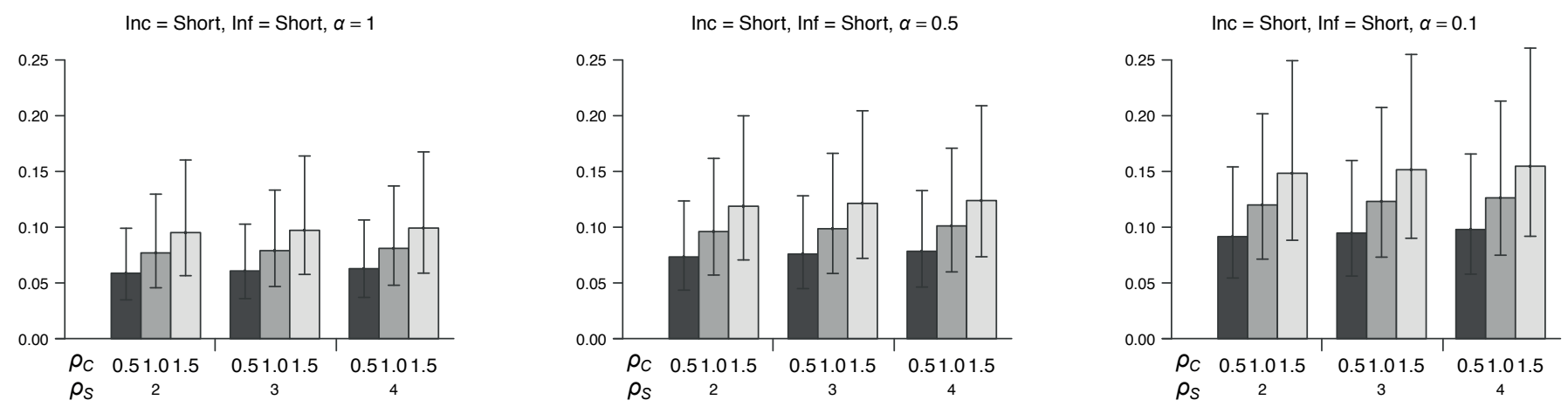

Inc: incubation period; Inf: infectious period.

The vertical axes are the estimates of basic reproductive number $\left(R_{o}\right)$. The estimates are stratified by the duration of the incubation and infectious periods (duration settings are given in Table 1), the relative contact frequency between a case and their household members within a hospital compared with within the household $(a)$, and the relative contact frequencies in schools and the community, $\rho_{s}$ and $\rho_{c}$. Bars represent $95 \%$ confidence intervals.

gain much human-to-human transmissibility between the two waves.

Two of the families in the second wave may have had multiple index cases, since symptom onsets in the first and second cases were only two days apart, and both cases were believed to have had exposure to live poultry. A sensitivity analysis was performed assuming the first two cases in each of these two families were both index cases. This assumption of multiple index cases removes the short serial intervals, leading to a longer mean serial interval of 8.4 days ( $95 \% \mathrm{Cl}: 6.8$ to 9.6). In the setting of medium-length incubation and infectious periods and $\mathrm{a}=1$, estimates for the SAR and $R_{0}$ are slightly lowered, to $1.2 \%$ (95\% Cl: 0.68 to 2.1 ) and 0.067 (95\% Cl: 0.038 to 0.12 ), respectively. The differences in transmissibility between waves and in the risk of infection between adults and children further diminish with the odds ratios, to 1.0 ( $95 \% \mathrm{Cl}: 0.29$ to 3.48 ) and 0.93 (95\% Cl: 0.25 to 3.51), respectively. 
Estimates of odds ratios of within-household secondary transmission with regard to the wave indicator (second wave to first wave), sex (men to women) and age group (adults to children) a based on household clusters of avian influenza A(H7N9) cases in humans, China, February-May 2013 (113 households, 118 cases) and October 2013-March 2014 (181 households, 190 cases)

\begin{tabular}{|c|c|c|c|c|c|}
\hline \multirow{3}{*}{$\begin{array}{l}\text { Incubation } \\
\text { period }\end{array}$} & \multirow{3}{*}{$\begin{array}{l}\text { Infectious } \\
\text { period }\end{array}$} & \multirow{3}{*}{ Value } & \multicolumn{3}{|c|}{ Relative contact frequency within hospital vs within household, $\alpha$} \\
\hline & & & 1.0 & 0.5 & 0.1 \\
\hline & & & Estimate $(95 \% \mathrm{Cl})$ & Estimate $(95 \% \mathrm{Cl})$ & Estimate $(95 \% \mathrm{Cl})$ \\
\hline \multirow{9}{*}{ Short } & \multirow{3}{*}{ Short } & Wave & $1.56(0.45$ to 5.39$)$ & $1.57(0.46$ to 5.39$)$ & $1.57(0.46$ to 5.39$)$ \\
\hline & & Sex & $2.65(0.78$ to 9.0$)$ & $2.63(0.78$ to 8.89$)$ & $2.60(0.77$ to 8.80$)$ \\
\hline & & Age & $0.51(0.15$ to 1.74$)$ & $0.49(0.15$ to 1.69$)$ & $0.48(0.14$ to 1.65$)$ \\
\hline & \multirow{3}{*}{ Medium } & Wave & $1.30(0.40$ to 4.27$)$ & $1.31(0.40$ to 4.27$)$ & $1.31(0.40$ to 4.26$)$ \\
\hline & & Sex & $2.24(0.69$ to 7.24$)$ & $2.21(0.69$ to 7.12$)$ & 2.18 (0.68 to 7.0$)$ \\
\hline & & Age & $0.66(0.20$ to 2.18$)$ & $0.64(0.19$ to 2.10$)$ & 0.62 (0.19 to 2.01) \\
\hline & \multirow{3}{*}{ Long } & Wave & $1.30(0.40$ to 4.26$)$ & $1.30(0.40$ to 4.25$)$ & 1.31 (0.40 to 4.23) \\
\hline & & Sex & 2.23 (0.69 to 7.20$)$ & $2.20(0.68$ to 7.07$)$ & 2.17 (0.68 to 6.92) \\
\hline & & Age & $0.66(0.20$ to 2.17$)$ & 0.64 (0.19 to 2.08$)$ & 0.61 (0.19 to 1.98$)$ \\
\hline \multirow{9}{*}{ Medium } & \multirow{3}{*}{ Short } & Wave & $1.30(0.40$ to 4.29$)$ & $1.31(0.40$ to 4.31$)$ & $1.32(0.40$ to 4.32$)$ \\
\hline & & Sex & 2.25 (0.69 to 7.29$)$ & $2.23(0.69$ to 7.21$)$ & $2.21(0.68$ to 7.15$)$ \\
\hline & & Age & $0.66(0.20$ to 2.20$)$ & $0.64(0.20$ to 2.13$)$ & 0.63 (0.19 to 2.07$)$ \\
\hline & \multirow{3}{*}{ Medium } & Wave & $1.30(0.40$ to 4.27$)$ & $1.31(0.40$ to 4.27$)$ & $1.31(0.40$ to 4.26$)$ \\
\hline & & Sex & $2.24(0.69$ to 7.26$)$ & $2.22(0.69$ to 7.14$)$ & $2.19(0.68$ to 7.02$)$ \\
\hline & & Age & 0.66 (0.20 to 2.19) & 0.64 (0.19 to 2.10$)$ & 0.62 (0.19 to 2.01$)$ \\
\hline & \multirow{3}{*}{ Long } & Wave & $1.30(0.40$ to 4.26$)$ & $1.30(0.40$ to 4.25$)$ & $1.30(0.40$ to 4.23$)$ \\
\hline & & Sex & 2.23 (0.69 to 7.22$)$ & 2.21 (0.69 to 7.09$)$ & $2.17(0.68$ to 6.94$)$ \\
\hline & & Age & 0.66 (0.20 to 2.17$)$ & 0.64 (0.19 to 2.08$)$ & 0.61 (0.19 to 1.98$)$ \\
\hline \multirow{9}{*}{ Long } & \multirow{3}{*}{ Short } & Wave & $1.30(0.40$ to 4.29$)$ & $1.31(0.40$ to 4.30$)$ & $1.32(0.40$ to 4.31$)$ \\
\hline & & Sex & $2.26(0.70$ to 7.32$)$ & 2.24 (0.69 to 7.24$)$ & $2.22(0.69$ to 7.18$)$ \\
\hline & & Age & 0.66 (0.20 to 2.20$)$ & $0.65(0.20$ to 2.13$)$ & 0.63 (0.19 to 2.07$)$ \\
\hline & \multirow{3}{*}{ Medium } & Wave & $1.30(0.39$ to 4.27$)$ & $1.30(0.40$ to 4.26$)$ & 1.31 (0.40 to 4.25) \\
\hline & & Sex & 2.25 (o.69 to 7.29 ) & $2.22(0.69$ to 7.16$)$ & $2.19(0.68$ to 7.04$)$ \\
\hline & & Age & 0.66 (0.20 to 2.19) & 0.64 (0.19 to 2.10$)$ & 0.62 (0.19 to 2.01) \\
\hline & \multirow{3}{*}{ Long } & Wave & $1.30(0.40$ to 4.26$)$ & $1.30(0.40$ to 4.24$)$ & $1.30(0.40$ to 4.22$)$ \\
\hline & & Sex & 2.24 (0.69 to 7.24$)$ & 2.21 (0.69 to 7.11$)$ & 2.18 (0.68 to 6.96$)$ \\
\hline & & Age & 0.66 (0.20 to 2.17$)$ & 0.64 (0.19 to 2.09$)$ & 0.61 (0.19 to 1.98$)$ \\
\hline
\end{tabular}

$\mathrm{Cl}$ : confidence interval.

Results are based on the assumption that the daily probability of infection by external sources (b) $=$ o (i.e. ignoring poultry-to-human transmission) and stratified by distributions of the incubation and infectious periods (duration settings are given in Table 1 ) and the relative contact frequency between a case and their household members within a hospital compared with within the household.

adults: 18 years or older; children: under 18 years.

Thus far, we have assumed $b=0$, i.e. all secondary cases were infected by the index cases. To assess how sensitive the estimates of SAR and $R_{0}$ are to the value of $b$ and to allow for the possibility of imported cases during each household outbreak, another set of analyses were conducted with $b=9.67 \times 10^{-5}$, the only non-zero MLE obtained when both the incubation and infectious periods are short. As expected, the estimates of both the SAR and $R_{\mathrm{o}}$ are slightly lowered (Table 4 ). The ranges of the point estimates are 1.1-2.0\% for the SAR and $0.06-0.11$ for $R_{0}$, compared with $1.3-2.2 \%$ and $0.07-0.12$, respectively, when $b=0$. Large values for $b$ would further reduce the estimates of SAR and $R_{0}$ but may not be reasonable assumptions as $b$ is generally much smaller than $p$ and the estimates of $p$ in our analyses are at the $10^{-3}$ scale.

\section{Discussion}

We found statistical evidence of human-to-human transmission of the avian influenza $A\left(\mathrm{H}_{7} \mathrm{~N} 9\right)$ virus, but it is clear that such transmission is not sustainable. Our estimate of the household SAR is below $2 \%$, and the estimate of $R_{0}=0.08$ is way below the threshold 1. In contrast, for seasonal human influenza, the estimates for the household SAR mostly range from $10 \%$ to $30 \%[22]$, and the $R_{\mathrm{o}}$ estimates vary from 1.1 to 1.6 
Estimates of secondary attack rate and basic reproductive number based on household clusters of avian H7N9 cases in humans, taking into account the probability of infection by external sources, China, February-May 2013 (113 households, 118 cases) and October 2013-March 2014 (181 households, 190 cases)

\begin{tabular}{|c|c|c|c|c|c|}
\hline \multirow{3}{*}{$\begin{array}{l}\text { Incubation } \\
\text { period }\end{array}$} & \multirow{3}{*}{$\begin{array}{l}\text { Infectious } \\
\text { period }\end{array}$} & \multirow{3}{*}{ Value } & \multicolumn{3}{|c|}{ Relative contact frequency within hospital vs within household, $\alpha$} \\
\hline & & & 1.0 & 0.5 & 0.1 \\
\hline & & & Estimate $(95 \% \mathrm{Cl})$ & Estimate $(95 \% \mathrm{Cl})$ & Estimate $(95 \% \mathrm{Cl})$ \\
\hline \multirow{6}{*}{ Short } & \multirow{2}{*}{ Short } & SAR & $1.1 \%(0.58$ to 2.0$)$ & $1.1 \%(0.61$ to 2.1$)$ & $1.2 \%(0.60$ to 2.2$)$ \\
\hline & & $R_{\mathrm{o}}$ & $0.061(0.033$ to 0.11$)$ & $0.064(0.034$ to 0.12$)$ & $0.065(0.034$ to 0.12$)$ \\
\hline & \multirow{2}{*}{ Medium } & SAR & $1.3 \%(0.71$ to 2.2$)$ & $1.4 \%(0.79$ to 2.5$)$ & $1.5 \%(0.79$ to 2.7$)$ \\
\hline & & $R_{\mathrm{o}}$ & 0.070 (0.040 to 0.12$)$ & $0.079(0.044$ to 0.14$)$ & $0.082(0.044$ to 0.15$)$ \\
\hline & \multirow{2}{*}{ Long } & SAR & $1.3 \%(0.74$ to 2.3$)$ & $1.6 \%(0.90$ to 2.8$)$ & $1.9 \%(1.0$ to 3.4$)$ \\
\hline & & $R_{\mathrm{o}}$ & $0.073(0.041$ to 0.13$)$ & $0.090(0.051$ to 0.16$)$ & $0.10(0.056$ to 0.19$)$ \\
\hline \multirow{6}{*}{ Medium } & \multirow{2}{*}{ Short } & SAR & $1.2 \%(0.69$ to 2.2$)$ & $1.3 \%(0.72$ to 2.3$)$ & $1.3 \%(0.75$ to 2.4$)$ \\
\hline & & $R_{0}$ & $0.069(0.038$ to 0.12$)$ & $0.072(0.040$ to 0.13$)$ & $0.075(0.042$ to 0.14$)$ \\
\hline & \multirow{2}{*}{ Medium } & SAR & $1.3 \%(0.73$ to 2.2$)$ & $1.5 \%(0.83$ to 2.6$)$ & $1.6 \%(0.90$ to 2.9$)$ \\
\hline & & $R_{\mathrm{o}}$ & $0.072(0.041$ to 0.13$)$ & $0.082(0.046$ to 0.15$)$ & $0.090(0.051$ to 0.16$)$ \\
\hline & \multirow{2}{*}{ Long } & SAR & $1.3 \%(0.72$ to 2.3$)$ & $1.6 \%(0.90$ to 2.8$)$ & $2.0 \%(1.1$ to 3.5$)$ \\
\hline & & $R_{\mathrm{o}}$ & $0.072(0.040$ to 0.13$)$ & $0.090(0.051$ to 0.16$)$ & $0.11(0.061$ to 0.20$)$ \\
\hline \multirow{6}{*}{ Long } & \multirow{2}{*}{ Short } & SAR & $1.3 \%(0.75$ to 2.3$)$ & $1.4 \%(0.80$ to 2.4$)$ & $1.5 \%(0.84$ to 2.5$)$ \\
\hline & & $R_{\mathrm{o}}$ & $0.073(0.042$ to 0.13$)$ & $0.078(0.045$ to 0.14$)$ & $0.082(0.047$ to 0.14$)$ \\
\hline & \multirow{2}{*}{ Medium } & SAR & $1.3 \%(0.73$ to 2.3$)$ & $1.5 \%(0.85$ to 2.6$)$ & $1.7 \%(0.97$ to 3.0$)$ \\
\hline & & $R_{\mathrm{o}}$ & $0.072(0.041$ to 0.13$)$ & $0.084(0.048$ to 0.15$)$ & $0.095(0.054$ to 0.17$)$ \\
\hline & \multirow{2}{*}{ Long } & SAR & $1.3 \%(0.72$ to 2.3$)$ & $1.6 \%(0.92$ to 2.8$)$ & $2.0 \%(1.2$ to 3.5$)$ \\
\hline & & $R_{0}$ & $0.072(0.040$ to 0.13$)$ & 0.091 (0.051 to 0.16$)$ & $0.11(0.065$ to 0.20$)$ \\
\hline
\end{tabular}

$\mathrm{Cl}$ : confidence interval; $R_{0}$ : basic reproductive number; SAR: secondary attack rate.

Results are based on the assumption that the daily probability of infection by external sources $(b)=9.67 \times 10^{-5}$ and are stratified by the duration of the incubation and infectious periods (duration settings are given in Table 1) and the relative contact frequency between a case and their household members within a hospital compared with within the household $(\alpha)$. Relative contact frequencies in schools and the community are set respectively to $\rho_{S}=3$ and $\rho_{C}=1$.

[31]. For past pandemic influenza strains, $R_{0}$ estimates are somewhat higher, e.g. from 1.3 to 1.7 for $A\left(\mathrm{H}_{1} \mathrm{~N}_{1}\right)$ pdmo9, 1.5 to 1.8 for $\mathrm{H}_{2} \mathrm{~N}_{2}$ and $\mathrm{H}_{3} \mathrm{~N}_{2}$ in the late $1950 \mathrm{OS}$ and $60 \mathrm{~s}$, and 1.8 to 2.4 for $\mathrm{H}_{1} \mathrm{~N}_{1}$ in 1918 [22,32]. Our $R_{0}$ estimate for $\mathrm{H}_{7} \mathrm{~N} 9$ is similar to a previous estimate of 0.10 [19]. However, our estimate is based on comprehensive household data covering two epidemic waves and partially accounts for heterogeneity in clustering pattern across age groups, whereas the previous estimate was made using only data from the first wave under the simplified but unrealistic assumption of random mixing of the whole population. Our estimate is relatively robust to uncertainty in the natural history of the disease but is somewhat sensitive to the relative transmission intensity in the community at large. Obtained via a Bayesian approach, the previous estimate had a credible interval of 0.01 to 0.49 , much wider than our Cls, even with the uncertainty in $\rho_{C}$ and $\rho_{S}$ factored in, and was sensitive to the prior distribution of $R_{0}$. In addition, we provide the first estimate for the household SAR, which could be useful in future simulation studies involving household transmission.

In the resampling-based testing of $p=0$, we implicitly assume that $b$ is constant over time. This assumption is unlikely to be true, as the exposure to zoonotic infection was substantially reduced when live poultry markets were closed in heavily affected areas from midApril to June in 2013 and from late January to February in 2014 to control the spread [33]. Due to the difficulty of the resampling-based test in handling covariates, we used an asymptotic test [34] to address this issue. The effect of market closure on $b$ has to be assumed known to implement this test, and we explored three values for the odds ratio (1.0, 0.5 and 0.1$)$ for market closure versus without closure. The value of 0.1 , or $90 \%$ reduction, is close to previous estimates [33]. The $\mathrm{p}$ values are all less than 0.001 , consistent with the statistical significance of human-to-human transmission. However, the significance level should not be overinterpreted, as the asymptotic test may not be suitable for sparse data [29].

We did not find statistical evidence for sex or age differences in the risk of human-to-human transmission, which could be due to the lack of power to detect such differences. Higher risk of poultry-to-human transmission in males was previously noticed during the first wave [6] and was also reflected in the male-to-female ratio of index case numbers in our household data: 
$88 / 37$ during the first wave and $124 / 65$ during the second. One hypothesis for such a difference between the sexes is that in the Yangtze River Delta, in particular Shanghai, where the first wave originated from, old men more frequently visit live poultry markets than women. In our analysis, male household contacts appeared to have a somewhat higher risk of infection than female contacts. If this sex difference was true, it might imply that the majority of non-index cases were infected by zoonotic rather than human sources. On the other hand, if there were truly no age difference, it implies that most non-index household cases were infected by human sources, or otherwise more adult non-index cases would have been observed in households, because adults were believed to be more prone to infection by poultry due to increased exposure $[6,30]$. The lack of age difference in human-to-human transmission is also consistent with the fact that most people were naive to this novel virus [35]. More household data in future outbreaks should be collected to re-examine the sex and age effects on the human-tohuman transmissibility of the virus.

Our analysis could be improved if we had genetic sequences and/or exposure and contact tracing information to help narrow the source of infection. Genetic linkage has been established in a couple of households included in our data [15-17]. However, similar viral genetic sequences do not necessarily imply humanto-human transmission, as the same virus could have been contracted from the same animal source. On the other hand, a certain level of heterogeneity in genetic sequences would imply impossible direct transmission pairs. Exposure and contact tracing information was obtained for some but not all cases in our study, but such data lack details and are subject to recall bias. Moreover, simultaneous testing of both human cases and possible animal sources would be helpful, but it is often difficult to trace the animal source, e.g. poultry purchased as food, or to coordinate between administrative units in charge of human health and those responsible for animal surveillance.

The human-to-human transmissibility of $\mathrm{H}_{7} \mathrm{Ng}$ remained not only limited but also temporally stable. We observed only a minor increase in the SAR estimates between the two successive waves, which is likely just a stochastic effect. However, some recent studies suggested genetic changes in the RNA segments of NS, NP and PB of the viral samples in Guangdong Province of southern China during the second wave as compared with sequences in eastern and central China from the first wave [36]. In addition, these segments are similar to the influenza $\mathrm{A}\left(\mathrm{H}_{9} \mathrm{~N}_{2}\right)$ viruses circulating in the same province, giving rise to concern about reassortment of $\mathrm{H}_{7} \mathrm{~N} 9$ with viruses that could lead to more efficient human-to-human transmission. Therefore, the efforts on collecting and analysing household transmission data should be continued in addition to the routine surveillance and contact tracing. Should human $\mathrm{H}_{7} \mathrm{~N} 9$ transmissibility increase to the level of that of seasonal human influenza, it would be very important to deploy antivirals agents and vaccines in areas where human cases are occurring. Human $\mathrm{H}_{7} \mathrm{~N}$ 9-inactivated vaccines are being developed by government agencies and private pharmaceutical companies [37]. These vaccines are currently in phase I and II safety and immunogenicity trials. The use of antiviral agents and vaccines needs to be incorporated into a comprehensive programme for the assessment of transmissibility and intervention effectiveness.

\section{Acknowledgements}

YY, EK, DC, IL and MH were partially supported by the National Institute of General Medical Sciences MIDAS grant U54-GM111274; YY, IL and MH were also partially supported by the National Institute of Allergy and Infectious Diseases (NIAID) grant R37-Alo32042. EK was also supported by NIAID grant K99/Roo Alo95302. ZF and WC were partially supported by the Ministry of Science and Technology of China grant KJYJ-2013-01-02.

\section{Conflict of interest}

None declared.

\section{Authors' contributions}

Yang Yang, Ira Longini, Zijian Feng and Wuchun Chao conceived the study and evaluated study results. Yang Yang, Liqun Fang, and Ira Longini designed analysis plan. Zijian Feng, Yanping Zhang, Enfu Chen, Jiayu Hu and Fenyang Tang collected data. Liqun Fang and Maijuan Ma provided further data support and verification. Yang Yang conducted data analysis. M. Elizabeth Halloran, Song Liang, Tom Britton and Eben Kenah evaluated study results. Yang Yang drafted the manuscript. Yang Yang, Ira Longini, Elizabeth Halloran, Zijian Feng, Wuchun Chao and Song Liang contributed to the final version. Ira Longini, Zijian Feng and Wuchun Cao share equal senior responsibility.

\section{References}

1. Lam TTY, Wang J, Shen Y, Zhou B, Duan L, Cheung C-L, et al. The genesis and source of the $\mathrm{H}_{7} \mathrm{~N} 9$ influenza viruses causing human infections in China. Nature. 2013;502(7470):241-4. http://dx.doi.org/10.1038/nature12515 PMID:23965623

2. Liu D, Shi W, Shi Y, Wang D, Xiao H, Li W, et al. Origin and diversity of novel avian influenza $\mathrm{A} \mathrm{H}_{7} \mathrm{~N} 9$ viruses causing human infection: phylogenetic, structural, and coalescent analyses. Lancet. 2013;381(9881):1926-32. http://dx.doi. org/10.1016/S0140-6736(13)60938-1 PMID:23643111

3. Xiong X, Martin SR, Haire LF, Wharton SA, Daniels RS, Bennett MS, et al. Receptor binding by an $\mathrm{H} 7 \mathrm{~N} 9$ influenza virus from humans. Nature. 2013;499(7459):496-9. http://dx.doi. org/10.1038/nature12372 PMID:23787694

4. Belser JA, Gustin KM, Pearce MB, Maines TR, Zeng H, Pappas $\mathrm{C}$, et al. Pathogenesis and transmission of avian influenza $A$ $\left(\mathrm{H}_{7} \mathrm{~N} 9\right)$ virus in ferrets and mice. Nature. 2013;501(7468):556-9. http://dx.doi.org/10.1038/nature12391 PMID:23842497

5. Richard M, Schrauwen EJA, de Graaf M, Bestebroer TM, Spronken MIJ, van Boheemen S, et al. Limited airborne transmission of $\mathrm{H}_{7} \mathrm{~N} 9$ influenza A virus between ferrets. Nature. 2013;501(7468):560-3. http://dx.doi.org/10.1038/ nature12476 PMID:23925116

6. Cowling BJ, Jin L, Lau EHY, Liao Q, Wu P, Jiang H, et al. Comparative epidemiology of human infections with avian influenza $\mathrm{A} \mathrm{H} 7 \mathrm{~N} 9$ and $\mathrm{H}_{5} \mathrm{~N}_{1}$ viruses in China: a population based study of laboratory-confirmed cases. Lancet. 2013;382(9887):129-37. http://dx.doi.org/10.1016/So1406736(13)61171-X PMID:23803488 
7. Yu H, Cowling BJ, Feng L, Lau EHY, Liao Q, Tsang TK, et al. Human infection with avian influenza $\mathrm{A} \mathrm{H} 7 \mathrm{~N} 9$ virus: an assessment of clinical severity. Lancet. 2013;382(9887):13845. http://dx.doi.org/10.1016/S0140-6736(13)61207-6 PMID:23803487

8. Gao HN, Lu HZ, Cao B, Du B, Shang H, Gan JH, et al. Clinical findings in 111 cases of influenza $A\left(\mathrm{H}_{7} \mathrm{Ng}\right)$ virus infection. $\mathrm{N}$ Engl J Med. 2013;368(24):2277-85. http://dx.doi.org/10.1056/ NEJMoa1305584 PMID:23697469

9. Dudley JP, Mackay IM. Age-specific and sex-specific morbidity and mortality from avian influenza $\mathrm{A}\left(\mathrm{H}_{7} \mathrm{~N} 9\right)$. J Clin Virol. 2013;58(3):568-70. http://dx.doi.org/10.1016/j.jcv.2013.09.004 PMID:24091087

10. Hope-Simpson RE. The role of season in the epidemiology of influenza. J Hyg (Lond). 1981;86(1):35-47. http://dx.doi. org/10.1017/So022172400068728 PMID:7462597

11. Hampson AW. Epidemiological data on influenza in Asian countries. Vaccine. 1999;17(Suppl 1):S19-23. http://dx.doi. org/10.1016/S0264-410X(99)00100-0 PMID:10471175

12. Chen E, Chen Y, Fu L, Chen Z, Gong Z, Mao H, et al. Human infection with avian influenza $\mathrm{A}(\mathrm{H} 7 \mathrm{~N} 9)$ virus re-emerges in China in winter 2013. Euro Surveill. 2013;18(43):20616. http://dx.doi.org/10.2807/1560-7917.ES2013.18.43.20616 PMID:24176616

13. Sutton TC, Finch C, Shao H, Angel M, Chen H, Capua I, et al. Airborne transmission of highly pathogenic $\mathrm{H}_{7} \mathrm{~N}_{1}$ influenza virus in ferrets. J Virol. 2014;88(12):6623-35. PMID:24696487

14. Halloran ME, Ferguson NM, Eubank S, Longini IM Jr, Cummings DA, Lewis B, et al. Modeling targeted layered containment of an influenza pandemic in the United States. Proc Natl Acad Sci USA. 2008;105(12):4639-44. http://dx.doi.org/10.1073/ pnas.0706849105 PMID:1833243

15. Qi X, Qian YH, Bao CJ, Guo XL, Cui LB, Tang FY, et al. Probable person to person transmission of novel avian influenza $A\left(\mathrm{H}_{7} \mathrm{~N} 9\right)$ virus in Eastern China, 2013: epidemiological investigation. BMJ. 2013;347:f4752. http://dx.doi.org/10.1136/ bmj.f4752 PMID:23920350

16. Hu J, Zhu Y, Zhao B, Li J, Liu L, Gu K, et al. Limited humanto-human transmission of avian influenza $\mathrm{A}\left(\mathrm{H}_{7} \mathrm{Ng}\right.$ ) virus, Shanghai, China, March to April 2013. Euro Surveill. 2014;19(25):20838. http://dx.doi.org/10.2807/1560-7917. ES2014.19.25.20838 PMID:24993556

17. Xiao XC, Li KB, Chen ZQ, Di B, Yang ZC, Yuan J, et al. Transmission of avian influenza $A\left(\mathrm{H}_{7} \mathrm{~N} 9\right)$ virus from father to child: a report of limited person-to-person transmission, Guangzhou, China, January 2014. Euro Surveill. 2014;19(25). pii: 20837. http://dx.doi.org/10.2807/1560-7917. ES2014.19.25.20837 PMID:24993555

18. Li Q, Zhou L, Zhou M, Chen Z, Li F, Wu H, et al. Epidemiology of human infections with avian influenza $\mathrm{A}\left(\mathrm{H}_{7} \mathrm{~N} 9\right)$ virus in China. N Engl J Med. 2014;370(6):520-32. http://dx.doi.org/10.1056/ NEJMoa1304617 PMID:23614499

19. Chowell G, Simonsen L, Towers S, Miller MA, Viboud C. Transmission potential of influenza $\mathrm{A} / \mathrm{H}_{7} \mathrm{~N} 9$, February to May 2013, China. BMC Med. 2013;11(1):214. http://dx.doi. org/10.1186/1741-7015-11-214 PMID:24083506

20. Chen Y, Liang W, Yang S, Wu N, Gao H, Sheng J, et al. Human infections with the emerging avian influenza $\mathrm{A} H 7 \mathrm{~N}$ 9 virus from wet market poultry: clinical analysis and characterisation of viral genome. Lancet. 2013;381(9881):1916-25. http://dx.doi. org/10.1016/S0140-6736(13)60903-4 PMID:23623390

21. Huang Y, Xu K, Ren DF, Ai J, Ji H, Ge AH, et al. Probably longer incubation period for human infection with avian influenza A ( $\left.\mathrm{H}_{7} \mathrm{~N} 9\right)$ virus in Jiangsu province, China, 2013. Epidemiol Infect. 2014;142(12):2647-53. http://dx.doi.org/10.1017/ So950268814000272

22. Yang Y, Sugimoto JD, Halloran ME, Basta NE, Chao DL, Matrajt $L$, et al. The transmissibility and control of pandemic influenza A ( $\left.\mathrm{H}_{1} \mathrm{~N}_{1}\right)$ virus. Science. 2009;326(5953):729-33. http://dx.doi. org/10.1126/science.1177373 PMID:19745114

23. Clinical signs and symptoms of influenza. http://www.cdc. gov/flu/professionals/acip/clinical.htm; last accessed on $8 / 15 / 2014 \cdot$ /eref $\rangle$

24. 〈jrn>24. Murphy BR, Rennels MB, Douglas RG Jr, Betts RF, Couch RB, Cate TR Jr, et al. Evaluation of influenza A/Hong Kong/123/77 ( $\left.\mathrm{H}_{1} \mathrm{~N}_{1}\right)$ ts-1A2 and cold-adapted recombinant viruses in seronegative adult volunteers. Infect Immun. 1980;29(2):348-55. PMID:7216417

25. To KK, Chan KH, Li IW, Tsang TY, Tse H, Chan JF, et al. Viral load in patients infected with pandemic $\mathrm{H}_{1} \mathrm{~N}_{1} 2009$ influenza A virus. J Med Virol. 2010;82(1):1-7. http://dx.doi.org/10.1002/ jmv.21664 PMID:19950247

26. Li IW, Hung IF, To KK, Chan KH, Wong SS, Chan JF, et al. The natural viral load profile of patients with pandemic 2009 influenza $A\left(\mathrm{H}_{1} \mathrm{~N}_{1}\right)$ and the effect of oseltamivir treatment.
Chest. 2010;137(4):759-68. http://dx.doi.org/10.1378/ chest.09-3072 PMID:20061398

27. Mok CKP, Lee HHY, Chan MCW, Sia SF, Lestra M, Nicholls JM, et al. Pathogenicity of the novel $\mathrm{A} / \mathrm{H}_{7} \mathrm{~N} 9$ influenza virus in mice. MBio. 2013;4(4). pii: e00362-13. http://dx.doi.org/10.1128/ mBio.00362-13 PMID:23820393

28. Yang Y, Longini IM Jr, Halloran ME. Design and evaluation of prophylactic interventions using infectious disease incidence data from close contact groups. J R Stat Soc Ser C Appl Stat. 2006;55(3):317-30. http://dx.doi.org/10.1111/j.14679876.2006.00539.x PMID:22457545

29. Yang Y, Longini IM, Halloran ME. A resampling-based test to detect person-to-person transmission of infectious disease. Ann Appl Stat. 2007;1(1):211-28. http://dx.doi.org/10.1214/07AOAS105 PMID:19436773

30. Liao Q, Lam WT, Leung GM, Jiang C, Fielding R. Live poultry exposure, Guangzhou, China, 2006. Epidemics. 2009;1(4):20712. http://dx.doi.org/10.1016/j.epidem.2009.09.002 PMID:21352767

31. Basta NE, Halloran ME, Matrajt L, Longini IM Jr. Estimating influenza vaccine efficacy from challenge and communitybased study data. Am J Epidemiol. 2008;168(12):1343-52. http://dx.doi.org/10.1093/aje/kwn259 PMID:18974084

32. Mills CE, Robins JM, Lipsitch M. Transmissibility of 1918 pandemic influenza. Nature. 2004;432(7019):904-6. http:// dx.doi.org/10.1038/nature03063 PMID:15602562

33. Yu H, Wu JT, Cowling BJ, Liao Q, Fang VJ, Zhou S, et al. Effect of closure of live poultry markets on poultry-toperson transmission of avian influenza $\mathrm{A} \mathrm{H}_{7} \mathrm{~N} 9$ virus: an ecological study. Lancet. 2014:383(9916):541-8. http://dx.doi. org/10.1016/S0140-6736(13)61904-2 PMID:24183056

34. Self SG, Liang KY. Asymptotic properties of maximum likelihood estimators and likelihood ratio tests under nonstandard conditions. J Am Stat Assoc. 1987;82(398):605-10. http://dx.doi.org/10.1080/01621459.1987.10478472

35. Bai T, Zhou J, Shu Y. Serologic study for influenza A $(\mathrm{H} 7 \mathrm{Ng})$ among high-risk groups in China. N Engl J Med. 2013;368(24):2339-40. http://dx.doi.org/10.1056/ NEJMC1305865 PMID:23718151

36. Lu J, Wu J, Guan D, Yi L, Zeng X, Zou L, et al. Genetic changes of reemerged influenza $A\left(\mathrm{H}_{7} \mathrm{~N}\right.$ 9) viruses, China. Emerg Infect Dis. 2014:20(9):1582-3. http://dx.doi.org/10.3201/eid2009.140250 PMID:25153821

37. National Institutes of Health (NIH). NIH begins testing $\mathrm{H}_{7} \mathrm{~N}_{9}$ avian influenza vaccine candidate. Bethesda, MD; 18 Sep 2013. [Accessed 31 Dec 2014]. Available from: http://www.nih.gov/ news/health/sep2013/niaid-18.htm 